Special Issue of the 6th International Congress \& Exhibition (APMAS2016), Maslak, Istanbul, Turkey, June 1-3, 2016

\title{
Effect of Heat Treatment and Pulse Plasma Process on Surface Properties of Steels
}

\author{
Y.Y. ÖZBEK* \\ Sakarya University, Engineering Faculty, Department of Metallurgical and Materials Engineering, \\ Esentepe Campus, 54187, Sakarya, Turkey

\begin{abstract}
The aim of this study was to examine the effect of heat treatment and pulse plasma treatment on surface properties of AISI 1050 steel used in this work. Firstly, the heat treatment was applied to samples for $2 \mathrm{~h}$ at $860{ }^{\circ} \mathrm{C}$. The quenching media were selected as water and oil and air cooling was applied. The tempering process was applied at different temperatures like $600{ }^{\circ} \mathrm{C}, 500{ }^{\circ} \mathrm{C}, 400{ }^{\circ} \mathrm{C}$ and $100^{\circ} \mathrm{C}$. Having completed the heat treatment, the pulse plasma process was applied to all samples. The surfaces were investigated with optic microscope, scanning electron microscopy, and X-ray diffraction analyses. The hardness testing was done for all samples from surface to center. The mechanical properties were improved after pulse plasma treatment.
\end{abstract}

DOI: 10.12693/APhysPolA.131.182

PACS/topics: 81.65.Lp

\section{Introduction}

Recently, number of surface modification techniques, such as high current pulse beam, laser, pulse plasma, plasma nitriding, and shot peening were reported to provide the improved superficial parameters (reduced surface roughness, increased hardness, and compressive residual stresses) responsible for the lowering of the stress concentration in surface topography and sub-surface microstructure [1-3]. Especially, pulse plasma has been proved to be a powerful tool for surface treatment of materials [2-5].

Pulse plasma technique induces modification by causing the change of morphology on treated surface of the material. When irradiated by pulse treatment, the near surface layer of targets undergoes a rapid melt and solidification with heating and cooling rates typically in the rank of $10^{7}-10^{10} \mathrm{~K} / \mathrm{s}$. These rates can initiate mixing, rapid diffusion and formation of amorphous or microcrystalline surface layers. Hence, the mechanical properties of material surfaces can be improved $[5,6]$.

In this work, the AISI 1050 steel was used. The steel is a medium carbon, medium tensile steel with good strength and toughness. The steel is used for the manufacture of forged shafts and gears and for a wide range of applications that can make use of its good combination of mechanical properties $[4,5]$. The heat treatment is carried out on this grade to render it suitable for machining and to impart it to specified mechanical properties. Then, the pulse plasma surface treatment of heat treated steel was conducted with efforts to investigate the surface modification. The different analyses techniques were applied to examine the samples after pulse plasma process.

*e-mail: yyarali@sakarya.edu.tr

\section{Experimental procedure}

The AISI 1050 steel was used in this work and its chemical composition was given in Table I. The samples were subjected to heat treatment at $860^{\circ} \mathrm{C}$. After the heat treatment, the samples were subsequently quenched in water, air, and oil. These samples were further heat treated followed by tempering at $600^{\circ} \mathrm{C}, 500^{\circ} \mathrm{C}, 400^{\circ} \mathrm{C}, 100^{\circ} \mathrm{C}$ temperature. Then, the pulse plasma process was applied to all samples. The process parameters of pulse were seen in Table II.

TABLE I

The chemical composition of AISI 1050 steel.

\begin{tabular}{c|c|c|c|c|c|c}
\hline \hline $\mathrm{C}$ & $\mathrm{Si}$ & $\mathrm{Mn}$ & $\mathrm{Cr}$ & $\mathrm{P}$ & $\mathrm{S}$ & $\mathrm{Fe}$ \\
\hline 0.48 & 0.25 & 0.61 & 0.455 & 0.040 & 0.050 & bal.
\end{tabular}

TABLE II

The process parameters of samples; distance nozzle $50 \mathrm{~mm}$, electrode - W, 10 pulses, battery capacity $800 \mathrm{mF}$.

\begin{tabular}{c|c|c}
\hline \hline No. & $\begin{array}{c}\text { Quenching after } \\
\text { heat treatment }\end{array}$ & $\begin{array}{c}\text { Tempering } \\
\text { temperature }\left[{ }^{\circ} \mathrm{C}\right]\end{array}$ \\
\hline 1 & air & 100 \\
2 & air & 400 \\
3 & air & 500 \\
4 & air & 600 \\
5 & water & 100 \\
6 & water & 400 \\
7 & water & 500 \\
8 & water & 600 \\
9 & oil & 100 \\
10 & oil & 400 \\
11 & oil & 500 \\
12 & oil & 600
\end{tabular}


The samples have been examined under optic microscope (Zeiss), scanning electron microscopy (SEM) (JEOL JSM-6500F and Cambridge Instrument) and X-ray diffraction (XRD) analyses (Rigaku) techniques. The hardness distribution on the cross-section of the treated layer was obtained by using Zeiss micro hardness tester with a load of $25 \mathrm{~g}$ and a loading time of $15 \mathrm{~s}$.

\section{Results and discussion}

Figure 1a,b shows us the micrograph of samples heat treated and quenched in air and oil, respectively. The ferrite and perlite structures were seen on surface. Slower cooling rates (in air) led to larger prior austenite grain size, larger amounts of soft ferrite and wider interlamellar spacing between cementite $\left(\mathrm{Fe}_{3} \mathrm{C}\right)$ plates of pearlite [6]. The initial microstructure 1050 steel was composed mainly of low carbon martensite laths and dispersed precipitation phases, which can be observed in Fig. 1a,b. The different cooling media was applied before

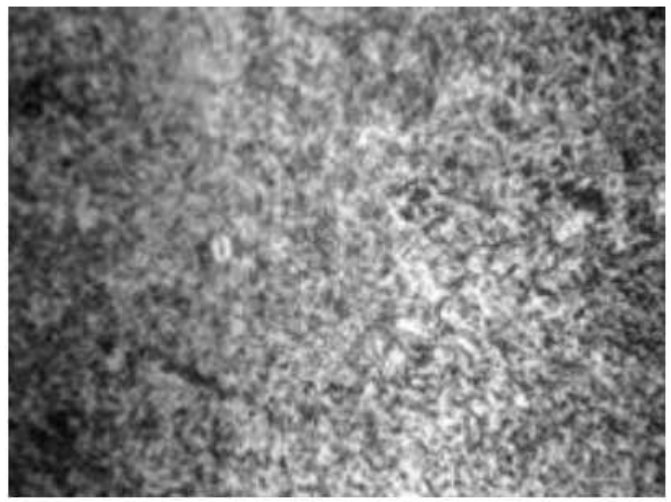

(a)

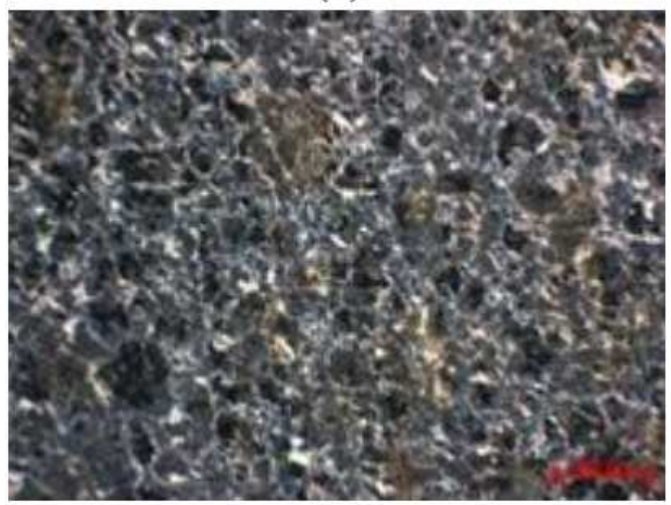

(b)

Fig. 1. The optic micrograph of samples before pulse plasma treatment (heat treated): (a) sample 1, (b) sample 9.

the pulse plasma treatment. Figure $2 \mathrm{a}-\mathrm{c}$ shows samples quenched by air, water and oil and pulse plasma treated, respectively. The cooling rate increment affects (in air) the incubation time and by decreasing the cooling rate, the incubation period decreases in high austenite grain size samples [6]. The cooling rate affects the microstructure. After pulse plasma treatment, the cross-sectional morphology observation, a graded modified structure was formed $[6,7]$. The microstructure of the modified layer, which develops upon pulse plasma pure iron using certain pulse plasma potential, consists of a compound and a diffusion zone underneath the compound layer [8]. The compound layer can be identified easily under metallographic examination since it is dark white. Figure $2 \mathrm{a}-\mathrm{c}$ shows the same pulse plasma parameters, but different cooling rates. Therefore, the microstructure is different.

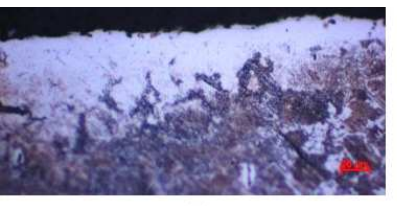

(a)

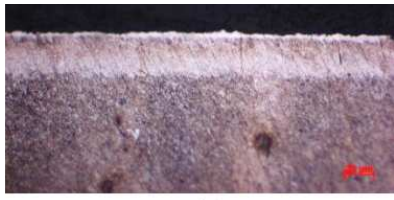

(b)

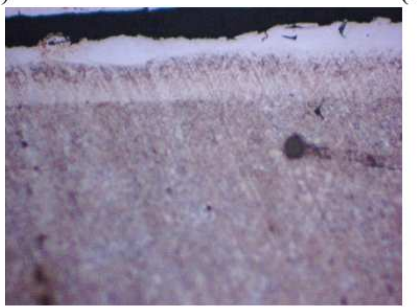

(c)

Fig. 2. The optic micrograph of the sample modified by pulse plasma treatment: (a) sample 1 , (b) sample 5 , (c) sample 9 .

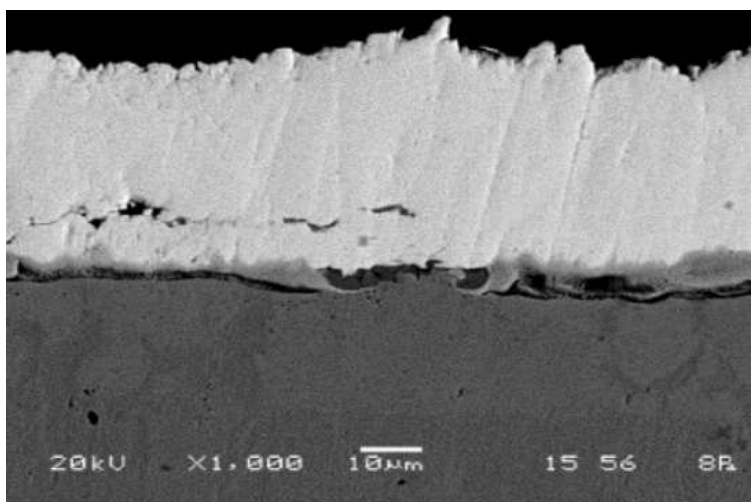

(a)

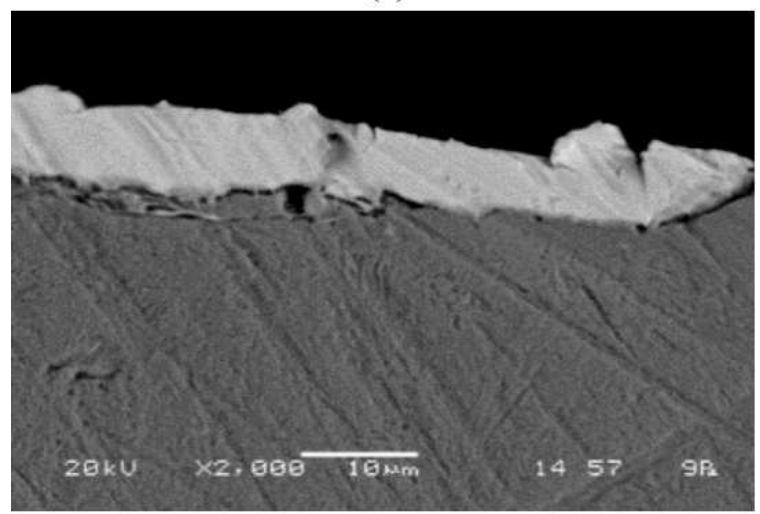

(b)

Fig. 3. SEM micrograph of (a) sample 3, (b) sample 6 . 
Figure 3a,b shows the SEM micrograph of samples modified with pulse plasma technique. The tempering temperature was different at samples. Microstructure changes occurred on surface consequences of a pulse plasma effect. The high energy density provides high temperature gradients, upon both heating and cooling of the work piece surface layer. The rapid solidification induced by pulse plasma leads to the formation of metastable structures, superfine grains and fine dendritic structure in the modified layer. Heating the work piece surface is realized also due to other types of effects: acoustic, mechanical, electromagnetic and beam [7, 8]. Also, the pulse plasma system modifies grain size of the layer [4]. The grain structure is very thin and dense in modified layer. The grain size variation is achieved via hot upsetting at different percentages of height reduction.

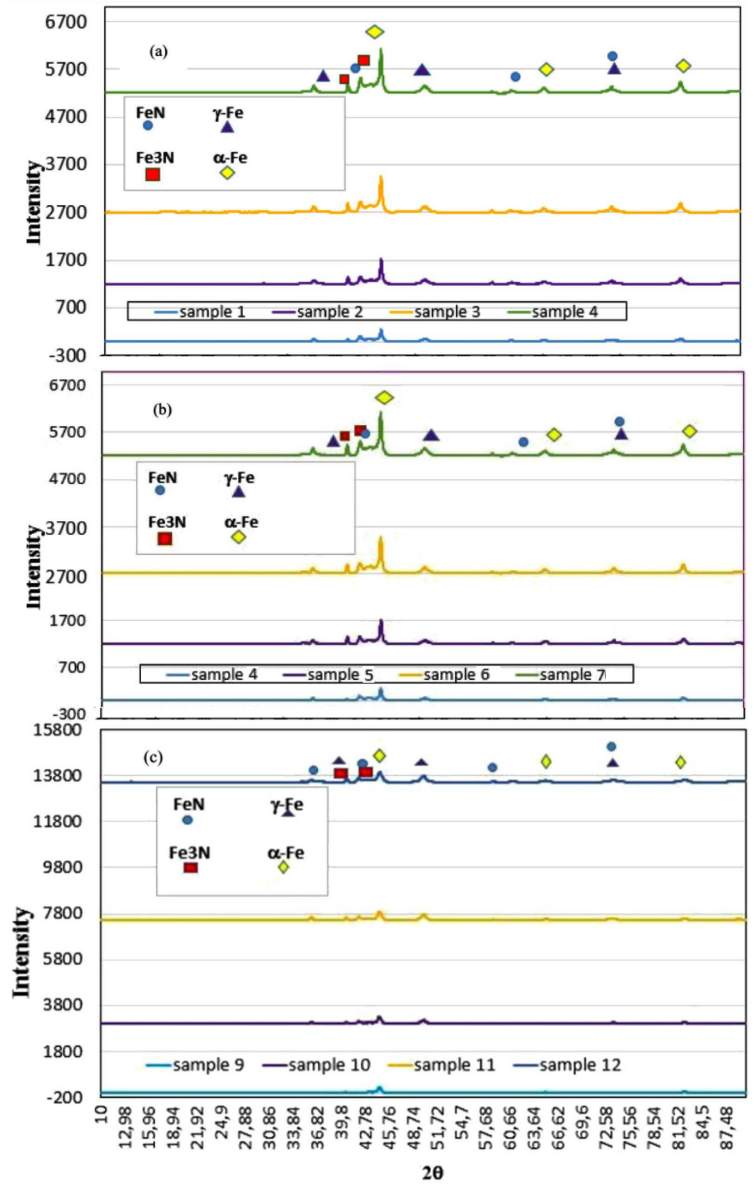

Fig. 4. XRD analyses of (a) samples 1-4, (b) samples 5-8, (c) samples 9-12.

The XRD analyses of samples produced by different pulse parameters were seen in Fig. $4 \mathrm{a}-\mathrm{c}$. After pulse plasma treatment, $\gamma$-Fe, $\mathrm{FeN}, \mathrm{Fe}_{3} \mathrm{~N}$ which are a new phase were formed. Depending on the nitrogen concentration in the gas mixture used during plasma treatment, the compound layer might be constituted by nitride phases [7]. The amount of phases for samples 1-4 in Fig. 4a (air + pulse plasma) are higher than samples 5-8 in Fig. $4 \mathrm{~b}$ (water+pulse plasma). The amount of phases was decreased in samples 9-12. This situation is given in Fig. 4c. However, the temperature of tempering treatment was affected to amount of phases (Fig. 4a-c) [6-9]. The attenuation was found to increase with increase in tempering temperature [6]. The phases and amount of these phases were very important to mechanical properties of surface. Because of this, these phases were very hard and increased hardness values of samples [8-11]. The variation of the parameters of heat treatment process and pulse plasma process resulted in different nitride layer characteristics; phase type, depth, hardness, hardness profile $[10,11]$. The relationship of the hardness between the samples is shown in Fig. $5 \mathrm{a}-\mathrm{c}$.
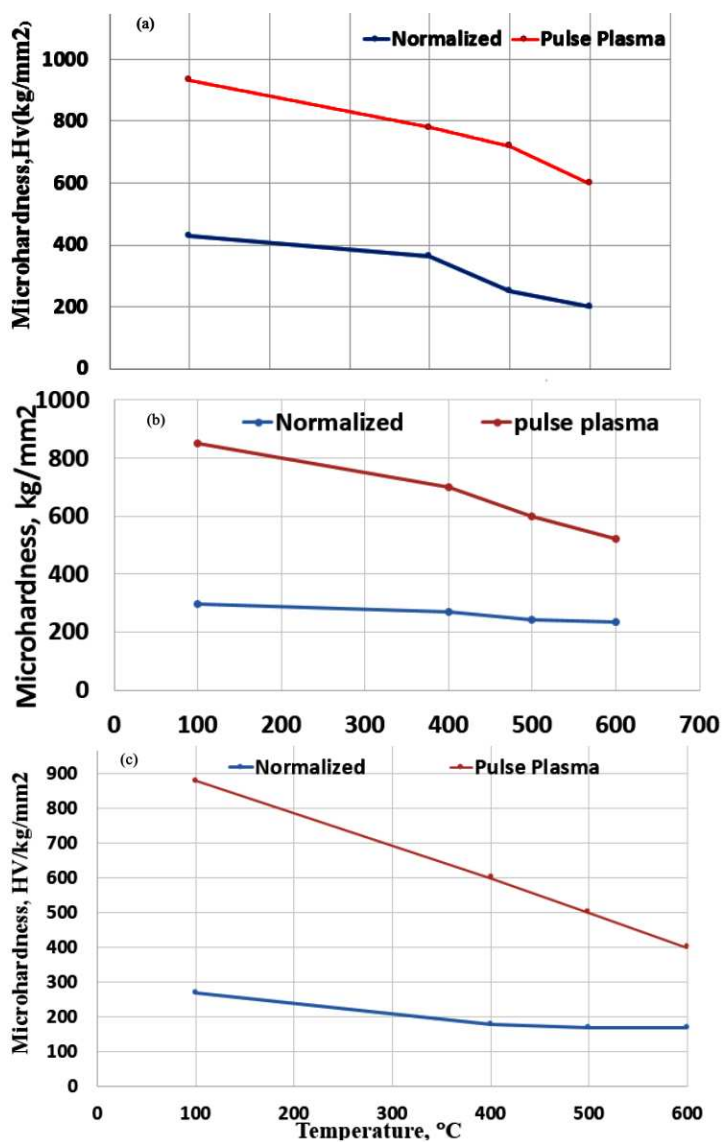

Fig. 5. The microhardness values of samples: (a) air + pulse plasma treatment, (b) water + pulse plasma treatment, (c) oil + pulse plasma treatment.

Prior to pulsed-plasma treatment, the initial hardness value of the specimens was recorded as $300 \mathrm{HV}$ and later its value was increased up to 700-970 HV. The hardness values increased 3-4 times after pulse plasma treatment [8]. The highest hardness values were seen for cooled at air + pulse plasma treated samples (Fig. 5a). The low hardness was seen to quench at oil + pulse plasma treated samples.

Significant increases in surface hardness were observed owing to the formation of modified layers on the new 
structured surface. The surface hardened layer depth at which the hardness reached the value of the core hardness. The structural defects, or disorder trappings, were easily formed at the high solidification rate induced by pulsed plasma. Another reason for the increase in the hardness values of the samples was the decreased grain size due to the increased cooling rate [11].

\section{Conclusion}

1. The surface treatment of 1050 steel was carried out in this work.

2. Firstly, the heat treatment was applied to the surface before pulse plasma treatment.

3. The different quenching media were applied like water and oil quenching and air cooling.

4. Pulse plasma treatment was applied to all samples. The modified layer consists of compound zone and diffusion zone occurred on surface after pulse plasma treatment.

5. The new and hard intermetallic phases such as FeN, $\mathrm{Fe}_{3} \mathrm{~N}$ were formed on surface after pulse plasma treatment.

6. The hardness values were increased after pulse plasma treatment. The highest hardness was seen on samples cooled with air and modified by pulse plasma.

\section{References}

[1] C.T. Kwok, F.T. Cheng, H.C. Man, Surf. Coat. Technol. 200, 3544 (2006).

[2] B.N. Mordyuk, G.I. Prokopenko, P.Yu. Volosevich, L.E. Matokhnyuk, A.V. Byalonovich, T.V. Popova, Mater. Sci. Eng. 659, 119 (2016).

[3] N.N. Cherenda, V.V. Uglov, A.K. Kuleshov, V.M. Astashynski, A.M. Kuzmitski, Vacuum 129, 170 (2016).

[4] Shengzhi Hao, Limin Zhao, Yanlong Zhang, Huihui Wang, Nucl. Instrum. Methods Phys. Res. B 356, 12 (2015).

[5] Alloy Information for AISI 1050 Carbon Steel, All Metals \& Forge Group.

[6] R. Prasad, S. Kumar, J. Mater. Process. Technol. 42, 51 (1994)

[7] Y.Y.Özbek, M. Durman, H. Akbulut, Tribol. Trans. $\mathbf{5 2}, 213$ (2009).

[8] Y.Y. Ozbek, C. Sarioglu, M. Durman, Vacuum 106, 11 (2014).

[9] J.S. Chen, C. Yu, H. Lu, J. Alloys Comp. 625, 224 (2015).

[10] C. Binder, T. Bendo, G. Hammes, A.N. Klei, J.D.B. de Mello, Wear 332-333, 995 (2015).

[11] Y.Y.Özbek, M. Durman, Mater. Technol. 49, 441 (2015). 